\title{
Expression and immunogenicity of six putative variable surface proteins in Mycoplasma mycoides subsp. mycoides SC
}

\author{
Carl Hamsten, ${ }^{1}$ Joakim Westberg, ${ }^{2}$ Göran Bölske, ${ }^{3}$ Roger Ayling, ${ }^{4}$ \\ Mathias Uhlén ${ }^{1}$ and Anja Persson ${ }^{1}$ \\ ${ }^{1}$ Department of Proteomics, School of Biotechnology, Royal Institute of Technology (KTH), \\ AlbaNova University Center, SE-106 91 Stockholm, Sweden \\ ${ }^{2}$ Department of Molecular Evolution, Evolutionary Biology Center, Uppsala University, SE-752 \\ 36 Uppsala, Sweden \\ ${ }^{3}$ Department of Bacteriology, National Veterinary Institute (SVA), SE-751 89 Uppsala, Sweden \\ ${ }^{4}$ The Mycoplasma Group, Veterinary Laboratories Agency (VLA), Addlestone, Surrey KT15 3NB, \\ UK
}

Correspondence

Anja Persson

anja@biotech.kth.se

Received 18 June 2007

Revised 1 November 2007

Accepted 8 November 2007
Variable surface protein Vmm and five Vmm-type proteins from Mycoplasma mycoides subsp. mycoides SC were analysed to determine whether these proteins are expressed in vivo in animals affected by contagious bovine pleuropneumonia (CBPP) and in vitro. Recombinant versions of these proteins were constructed and expressed in Escherichia coli after mutation of the TGA Trp codons to TGG. These proteins were then analysed by dot and Western blotting with sera from CBPP-affected cattle. Furthermore, affinity-purified polyclonal antibodies to the recombinant proteins were used in Western and colony blotting to look for expression of the putative Vmm-type proteins in cultured M. mycoides SC. This study demonstrates that immunoglobulins in CBPP sera recognize all putative $\mathrm{Vmm}$-type proteins tested, indicating that these proteins or their homologues are expressed by mycoplasmas during natural infections. Vmm and one of the putative Vmm-type proteins showed variable expression in vitro.

\section{INTRODUCTION}

Mycoplasma mycoides subsp. mycoides small colony type (M. mycoides SC) belongs to the class Mollicutes and is the causative agent of contagious bovine pleuropneumonia (CBPP), a severe disease affecting cattle. It is a notifiable disease that has to be reported to the World Organization for Animal Health (OIE). CBPP is a vast problem in Africa, where 18 countries reported 168 outbreaks in 2005 according to the OIE. The last outbreaks in Europe were in Italy (1993), Spain (1994) and Portugal (1999). The occurrence of CBPP in Asia has recently been reported from several countries, the latest being Kuwait in 2006 (World Animal Health Information Database, OIE).

The use of antibiotics, such as tylosin and tetracyclines, for treatment of CBPP is a much-debated subject. It has been considered ineffective and a potential promoter of silent

Abbreviations: $\mathrm{ABP}$, albumin binding protein; $\mathrm{CBPP}$, contagious bovine pleuropneumonia; FBS, fetal bovine serum; HRP, horseradish peroxidase; IMAC, immobilized metal affinity chromatography; pAb, polyclonal antibody.

A supplementary table listing the primers used in PCR, mutagenesis and DNA sequencing is available with the online version of this paper. carriers of the disease (Provost, 1996). However, newer antibiotics, such as danafloxacin, have been shown to reduce the spread of CBPP (Hübschle et al., 2006). The slaughter of infected herds still appears to be the most effective means to eradicate CBPP (Windsor \& Wood, 1998). This strategy has been used in Europe, but the disease has re-emerged in every decade of the 20th century (Nicholas et al., 2000). CBPP is endemic in much of subSaharan Africa, and eradication by mass slaughter would be too expensive and have severe consequences (Kusiluka \& Sudi, 2003; Windsor, 2000). In Botswana, CBPP was successfully eradicated in 1995 by a stamping-out strategy (Windsor \& Wood, 1998) which turned out to be directly correlated with increased malnutrition in children (Boonstra et al., 2001). Extensive vaccination campaigns seem to be the best option for Africa (March, 2004; Windsor, 2000). However, recent vaccination campaigns have given poor results due to weak vaccine effects (Nicholas et al., 2000; Thiaucourt et al., 1998, 2000). The vaccines currently in use are live strains with reduced pathogenicity, and the vaccine currently recommended by the OIE, strain T1-44, has disadvantages such as lack of long-term immunity (Kusiluka \& Sudi, 2003), late protection (1-3 months for primo-vaccination) 
(Thiaucourt et al., 1998) and poor protection in vaccine trials (Thiaucourt et al., 2000). Pathogenicity of T1-44 has also been shown (Mbulu et al., 2004).

Little is known of the pathogenicity of M. mycoides SC. No secreted toxins or surface receptors mediating adhesion or cellular responses in host tissues have been reported. There are factors associated with pathogenesis, although their precise functions are unclear. The galactan capsule, an oligosaccharide layer surrounding the cell membrane, appears to promote binding to host tissues, convey resistance to phagocytosis and have toxic effects, and may cause autoimmune responses by structural similarities to bovine pneumogalactan (Buttery et al., 1976; Nicholas \& Bashiruddin, 1995). Oxidative damage to the host tissue from hydrogen peroxide $\left(\mathrm{H}_{2} \mathrm{O}_{2}\right)$ produced by glycerol metabolism in M. mycoides SC (Miles et al., 1991) may contribute to CBPP lesions and has been described for other mycoplasma species (Tryon \& Baseman, 1992). Five immunogenic lipoproteins of $M$. mycoides SC, LppA (Cheng et al., 1996; Monnerat et al., 1999), LppB (Vilei et al., 2000), LppC (Pilo et al., 2003), LppQ (Abdo et al., 2000) and Vmm (Persson et al., 2002), have recently been described; however, their biological functions or possible roles in pathogenicity are, to our knowledge, still unknown.

Many mycoplasmas express surface proteins that undergo reversible changes to alter the antigenic repertoire at the cell and population levels (Citti \& Rosengarten, 1997; Razin et al., 1998), as an adaptation to evade the host immune response. Some variable surface proteins are involved in adhesion and immunomodulation (Le Grand et al., 1996; Sachse et al., 2000; Washburn et al., 1993). Only two variable surface proteins are known in $M$. mycoides SC (Gaurivaud et al., 2004; Persson et al., 2002). One of them, $\mathrm{Vmm}$, is a small lipoprotein of $17 \mathrm{kDa}$ whose expression is regulated at the transcriptional level by the number of (TA) $)_{n}$ repeats in the promoter spacer. Fourteen putative proteins with similar promoters are present in the M. mycoides SC genome (Westberg et al., 2004), and are referred to as Vmm-type proteins in this study.

The primary aim of this study was to investigate six of the Vmm-type proteins, to detect whether humoral immune responses are raised against them in vivo, if they are expressed in vitro and if the expression in vitro is variable. An additional aim was the development of an optimized scheme for high-throughput production of recombinant M. mycoides SC proteins in Escherichia coli. This would enable the screening of a large number of proteins for possible selection as target antigens for the development of diagnostic tests and recombinant vaccines.

\section{METHODS}

In silico analysis. Since several of the $14 \mathrm{Vmm}$-type proteins have high similarity to each other, a subset of six of them with low similarity to each other [determined by BLAST (Altschul et al., 1997)] were selected for this investigation. This subset included Vmm. Their peptide sequences were retrieved from the genome sequence at EMBL/GenBank/DDBJ entry BX293980 and analysed with TMHMM (Krogh et al., 2001) and SignalP (Nielsen et al., 1997; Nielsen \& Krogh, 1998) to identify transmembrane regions and signal peptide sequences, respectively, LipoP (Juncker et al., 2003) to predict lipoproteins, and InterProScan (Quevillon et al., 2005; Zdobnov \& Apweiler, 2001) to identify protein motifs.

Primer design and expression vector. PCR primers (see Supplementary Table S1 available with the online version of this paper) were designed to amplify the whole genes except the signal peptide sequences. Not I or AscI restriction sites were added to the $5^{\prime}$ end of the primers to allow directed insertion into the vector pAff $8 \mathrm{c}$ (Larsson et al., 2000). The reverse primer was biotinylated to enable solid-phase cloning, and a $3 \mathrm{C}$ protease cleavage site (not used in this study) was included in the forward primer handle to enable removal of the $\mathrm{His}_{6}$-albumin binding protein $(\mathrm{ABP})$ fusion tag of the recombinant protein. The $\mathrm{His}_{6}$ moiety of the fusion tag allows purification by immobilized metal affinity chromatography (IMAC), while the ABP (Nygren et al., 1988) enhances solubility and is immunopotentiating (Libon et al., 1999; Sjölander et al., 1997). Names of the recombinant proteins were derived from the corresponding ORF names (Westberg et al., 2004) from EMBL/ GenBank/DDBJ accession number BX293980.

M. mycoides SC strains. The M. mycoides SC type strain $\mathrm{PG} 1^{\mathrm{T}}$ was grown in F medium (Bölske, 1988). Genomic DNA was prepared and purified by proteinase $\mathrm{K}$ lysis and phenol:chloroform extraction. Total RNA was isolated with Trizol reagent (Life Technologies) from $200 \mathrm{ml}$ culture. For colony immunoblotting, M. mycoides SC strains $\mathrm{PG}^{\mathrm{T}}$ and $\mathrm{M} 223 / 90$, a pathogenic strain from Tanzania (Bölske et al., 1995), were grown on F medium agar plates.

Cloning. PCR was performed with $20 \mathrm{ng}$ template DNA using AmpliTaq DNA polymerase (Roche). The biotinylated PCR products were immobilized on Dynabeads M280-streptavidin paramagnetic beads (Dynal Biotech), and the bound PCR fragments were washed and cleaved with NotI (New England Biolabs). The buffer was replaced, and amplicons were released from the beads by AscI (New England Biolabs) digestion. Cleaved fragments were thereafter ligated into AscI/NotI-digested pAff8c plasmid using T4 DNA ligase (Fermentas) and the constructs were heat-shock transformed into E. coli strain BL21(DE3) cells (Novagen). Correct clone sequences were verified by sequencing. Single-stranded DNA template was generated using TempliPhi (GE Healthcare) prior to cycle sequencing with BigDye Terminator chemistry (Applied Biosystems). The sequencing reactions were analysed on an ABI PRISM 3700 sequencer (Applied Biosystems) and the data analysed using Sequencher software (Gene Codes). To substitute the mycoplasma TGA tryptophan codon with the E. coli TGG tryptophan codon, the QuikChange Multi Site-Directed Mutagenesis kit (Stratagene) was used. Plasmid preparations were made with the QIAprep Spin Miniprep kit (Qiagen).

RT-PCR. For expression analysis, RT-PCR was performed with the SuperScriptIII One-Step RT-PCR system with a Platinum Taq kit (Invitrogen) in 40 cycles. First-strand synthesis was performed with $1 \mu \mathrm{g}$ RNA and 15 pmol reverse primer at $50{ }^{\circ} \mathrm{C}$ for $30 \mathrm{~min}$.

Protein expression and purification. The recombinant proteins were expressed in E. coli BL21(DE3) and were purified by IMAC (Porath et al., 1975), as previously described (Steen et al., 2006). Purified samples were diluted from 6 to $1 \mathrm{M}$ urea with PBS $(2 \mathrm{mM}$ $\mathrm{NaH}_{2} \mathrm{PO}_{4}, 8 \mathrm{mM} \mathrm{Na}_{2} \mathrm{HPO}_{4}, 150 \mathrm{mM} \mathrm{NaCl}$ ) and were subsequently concentrated on a Vivapore $10 / 20$ concentrator (Vivascience) to a final volume of $1.5 \mathrm{ml}$. The protein concentrations were estimated 
with the bicinchoninic acid (BCA) assay, and the protein-50 assay (Agilent Technologies) was used to measure protein purity.

Antibodies and sera used in immunoblotting. Sera from 15 CBPP cases were used in this study. Four were from an outbreak in Botswana in the middle of the 1990s (G1-4), eight were from an outbreak in Namibia in May 2004 (1MUK15A-17MUK15A), one was an experimental infection from Namibia in 2001 (Exp. Inf.), one serum was from an outbreak in Tanzania in 1997 (PW227), and the final serum was from Kenya in 1998 (C102). For information on CBPP diagnostic test results, see Table 1. Fetal bovine serum (FBS; Gibco) and sera from five healthy Swedish cattle were used as negative controls. The sera were diluted 1:400 and 1:5000; both concentrations were used for each serum. They were blocked with an E. coli lysate and purified $\mathrm{His}_{6}-\mathrm{ABP}$ to prevent false signals due to interaction with residual E. coli proteins in the recombinant protein samples or to the $\mathrm{His}_{6}-\mathrm{ABP}$ tag which originates from streptococcal protein G. For the pre-adsorption experiments, $500 \mu \mathrm{g}$ of the six recombinant Vmm-type proteins was added to separate $1 \mathrm{ml}$ aliquots of serum 15MUK15A, prepared as described above.

Immunoblotting. The recombinant proteins were dot-blotted on nitrocellulose membranes (Bio-Rad Laboratories). The proteins $(2 \mu \mathrm{g}$ of each) as well as $\mathrm{His}_{6}-\mathrm{ABP}$ and a $\mathrm{PGl}^{\mathrm{T}}$ lysate for negative and positive controls were applied to the membrane. The membranes were blocked in PBST [PBS, $0.1 \%(\mathrm{v} / \mathrm{v})$ Tween] containing $25 \%(\mathrm{v} / \mathrm{v})$ horse serum (Gibco), followed by incubation with the bovine sera for $60 \mathrm{~min}$ at room temperature. Bound antibodies were detected with secondary goat anti-bovine IgG conjugated with horseradish peroxidase (HRP; $4 \mathrm{ng} \mathrm{ml}^{-1}$, Jackson Immunoresearch) and a chemiluminescent HRP substrate (Pierce Biotechnology). The membranes were developed in a ChemiDoc XRS detection system (Bio-Rad Laboratories). For Western blotting, $3 \mu \mathrm{g}$ recombinant proteins and/or a PG1 ${ }^{\mathrm{T}}$ lysate were first separated by SDS-PAGE in a $10-20 \%$ Tris/HCl Criterion Precast gel (Bio-Rad Laboratories) and then electrotransferred onto a nitrocellulose membrane (Bio-Rad Laboratories). Membranes were washed for $5 \mathrm{~min}$ in Tris-buffered saline (TBS; $1 \mathrm{mM}$ Tris, $15 \mathrm{mM} \mathrm{NaCl}, \mathrm{pH}$ 7.5) prior to staining with
Ponceau S (Sigma-Aldrich; 1:20 in MilliQ water). Blocking and serum screening were performed as described for dot blotting.

Immunizations and affinity purification of antibodies. Rabbits were immunized with the purified recombinant Vmm-type proteins in accordance with national guidelines (Swedish permit A84-02). The protocols for immunization and subsequent affinity purification of antibodies have been described previously (Nilsson et al., 2005). Briefly, the serum was depleted of antibodies reactive to the $\mathrm{His}_{6}-\mathrm{ABP}$ fusion tag, followed by enrichment of Vmm-type-protein-specific antibodies on an affinity column containing the recombinant proteins, and removal of all other antibodies. Collected antibody fractions were diluted $1: 1$ with $87 \%(\mathrm{v} / \mathrm{v})$ glycerol and $0.02 \%(\mathrm{v} / \mathrm{v})$ $\mathrm{NaN}_{3}$, and stored at $-20{ }^{\circ} \mathrm{C}$. Antibodies were named after the corresponding recombinant Vmm-type protein, e.g. polyclonal antibody (pAb) A117 was produced from recombinant protein R117, etc.

Colony immunostaining. Freshly grown mycoplasma colonies were transferred to nitrocellulose membranes and blocked in TBST [TBS, $0.05 \%(\mathrm{v} / \mathrm{v})$ Tween 20] containing $10 \%(\mathrm{v} / \mathrm{v})$ horse serum, followed by incubation with the affinity-purified pAbs (diluted 1:50, to 1.5$6.6 \mu \mathrm{g} \mathrm{ml}^{-1}$ ) for $1.5 \mathrm{~h}$ at room temperature. The $\mathrm{Vmm}$-specific $\mathrm{mAb}$ 5G1 (9 $\mu \mathrm{g} \mathrm{ml}^{-1}$ ) (Brocchi et al., 1993; Persson et al., 2002) was used as a positive control. Bound antibodies were detected with HRP-conjugated swine anti-mouse or goat anti-rabbit IgG (DakoCytomation) at a final concentration of $0.3 \mu \mathrm{g} \mathrm{ml}^{-1}$ using 4chloro-1-naphthol as substrate. The membranes were also stained with Ponceau S in order to identify negative colonies.

\section{RESULTS}

\section{Selection and in silico analysis of Vmm-type proteins}

The following protein genes were selected from the group of 15 Vmm-type proteins: MSC_0117, MSC_0364,

Table 1. Information on the sera used in immunoblotting experiments

CFT, complement fixation test; LAT, latex agglutination test; ND, not done.

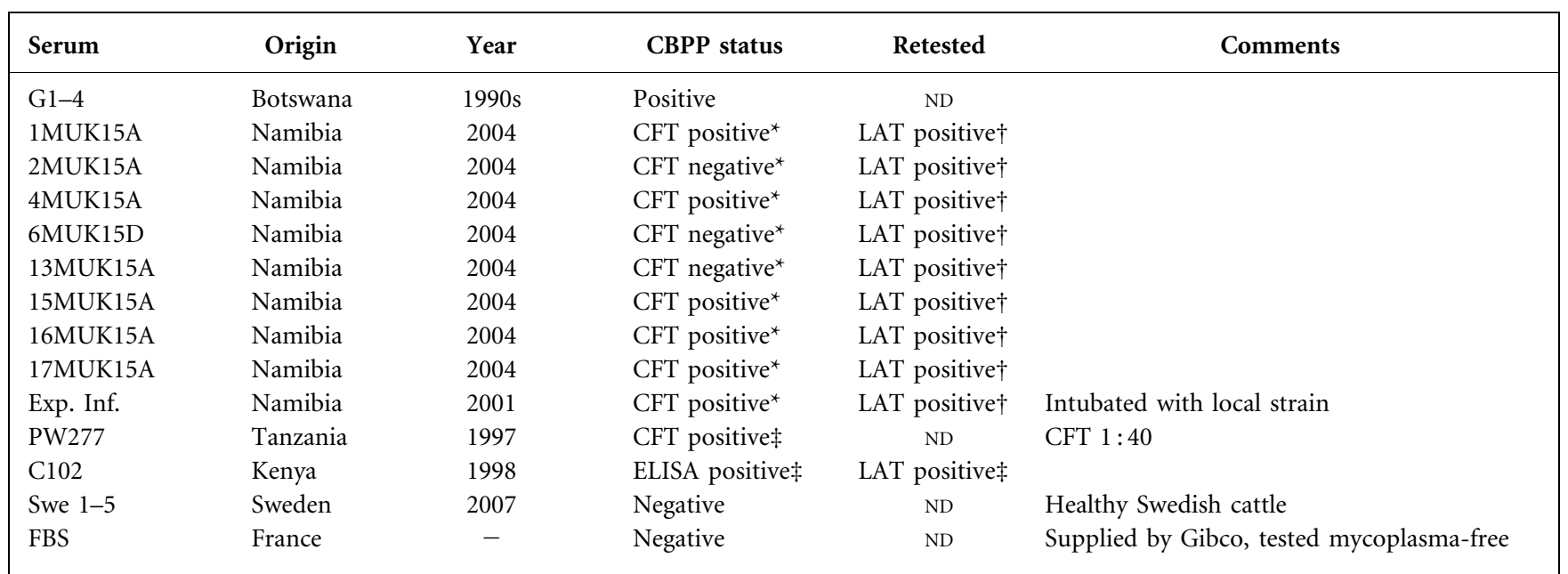

${ }^{\star}$ Hübschle et al. (2006).

$\nmid$ R. D. Ayling, unpublished data.

‡J. B. March, personal communication. 
MSC_0816, MSC_0847, MSC_1033 and vmm MSC_0390. Two proteins have an amino acid sequence similar to that of other proteins in M. mycoides SC according to BLAST comparisons in which the cut off was set to $80 \%$ identity for at least $80 \%$ of the sequence length. MSC_0816 is similar to MSC_0812, MSC_0815, MSC_0818 and MSC_0817, and MSC_1033 is homologous to MSC_1058. Sequence analysis with SignalP and TMHMM revealed a signal peptide for each protein, but no transmembrane regions were identified. Analysis with LipoP identified five proteins (excluding MSC_0816) as lipoproteins, which was consistent with the genomic annotation. Further analysis with InterProScan revealed no additional signature/pattern/motif information except for MSC_0816, which had InterPro motifs IPR005046 and IPR011889 in one region. Both motifs exist in families of proteins with unknown functions. Many of these proteins contain a tandem peptide repeat sequence of 25 or 26 amino acid residues (26 aa in MSC_0816, repeated twice) found in predicted surface proteins, often lipoproteins. The InterPro database contains 85 mollicute proteins that match the IPR005046 motif, of which several belong to the $M$. mycoides cluster. See Table 2 for a summary of in silico analysis.

\section{Cloning, in vitro mutagenesis and recombinant protein production}

In order to mimic the protein that is presented to the host immune system, recombinants were designed based on the full-length Vmm-type proteins, the signal peptide excepted. The average size of the signal peptide sequences that were excluded from each protein was 24 aa. Plasmids containing the gene fragment fused to a $\mathrm{His}_{6}-\mathrm{ABP}$ tag were constructed, and as many as nine TGA tryptophan codons were successfully substituted within a gene by using up to four codon substitutions per reaction. All six recombinant proteins were successfully expressed and purified, yielding protein concentrations of $0.5-4.9 \mathrm{mg} \mathrm{ml}^{-1}$ from $100 \mathrm{ml}$ culture.

\section{Humoral immune responses to the Vmm-type proteins in CBPP-diseased bovines}

In order to analyse whether native Vmm-type proteins are expressed in mycoplasmas during infection, the corresponding recombinant $\mathrm{Vmm}$-type proteins were subjected to dot and Western blotting against 15 bovine sera from four CBPP outbreaks, as well as five sera from healthy Swedish cattle and FBS as negative controls. Dot blotting was performed as an initial screening to allow analysis under non-denaturing conditions. An M. mycoides SC strain $\mathrm{PG} 1^{\mathrm{T}}$ lysate was used as a positive control, and the recombinant fusion partner $\mathrm{His}_{6}-\mathrm{ABP}$ and an E. coli whole-cell lysate were used as negative controls. The results (Fig. 1, Table 3) showed that all CBPP-positive sera contained antibodies that bound several recombinant proteins. Interestingly, the signal intensities varied between the sera. Generally, R816 was the predominant spot, while Vmm (R390) was absent or indistinguishable from signals seen in some of the negative controls, which is also demonstrated in the blot detected with a pool of all disease sera. There were no detectable false-positive signals due to the $\mathrm{His}_{6}-\mathrm{ABP}$ tag; however, a weak reaction to the E. coli lysate was seen for some sera. The five control sera from healthy bovines and FBS showed that there is no general cross-reactivity of serum IgGs to the recombinant proteins. A faint positive signal, considered to be noise, did occur for four of the control sera.

Additional dot-blotting experiments were performed to confirm the specificity of the serum antibodies. Serum 15 MUK15A was aliquoted into seven fractions, of which six were pre-adsorbed with individual recombinant proteins prior to the dot-blotting analysis, and the remainder were used untreated. An example of the results with R816 is given in Fig. 2. The protein-specific signals were significantly reduced in the pre-adsorbed sera compared to untreated sera, while all other proteins acted as controls and reacted with equal signal intensities, indicating specific binding of the serum antibodies to the recombinant proteins. Since a more concentrated $P G 1^{T}$

Table 2. Summary of bioinformatic analyses of the selected Vmm-type proteins

$\downarrow$ Represents the predicted cleavage site for the prolipoprotein signal peptidase.

\begin{tabular}{|c|c|c|c|c|c|c|}
\hline ORF & Promoter repeat & Lipobox & $\begin{array}{c}\text { Conserved } \\
\text { domain }\end{array}$ & $\begin{array}{l}\text { Molecular mass } \\
(\mathrm{kDa})^{\star}\end{array}$ & $\begin{array}{l}\text { Recombinant } \\
\text { protein ID }\end{array}$ & $\begin{array}{l}\text { Molecular mass, } \\
\text { recombinant protein }(\mathrm{kDa})\end{array}$ \\
\hline MSC_0117 & poly(TA) & VVA $\downarrow$ C & & 24.1 & R117 & 42.6 \\
\hline MSC_0364 & poly(TA) & TAS $\downarrow$ C & & 15.6 & R364 & 33.9 \\
\hline MSC_0390 & poly(TA) & VVA $\downarrow$ C & & 3.7 & R390 & 22.2 \\
\hline MSC_0816 & poly(A) & - & $\begin{array}{l}\text { IPR011889 } \\
\text { IPR005046 }\end{array}$ & 43.5 & $\mathrm{R} 816$ & 62.1 \\
\hline MSC_0847 & $\operatorname{poly}(A)$ & TVS $\downarrow \mathrm{C}$ & & 26.8 & R847 & 44.8 \\
\hline MSC_1033 & poly(TA) & $\mathrm{VIA} \downarrow \mathrm{C}$ & & 6.3 & R1033 & 25.6 \\
\hline
\end{tabular}

${ }^{\star}$ Molecular mass calculated using the Protparam tool (www.expasy.org), excluding signal peptides. 


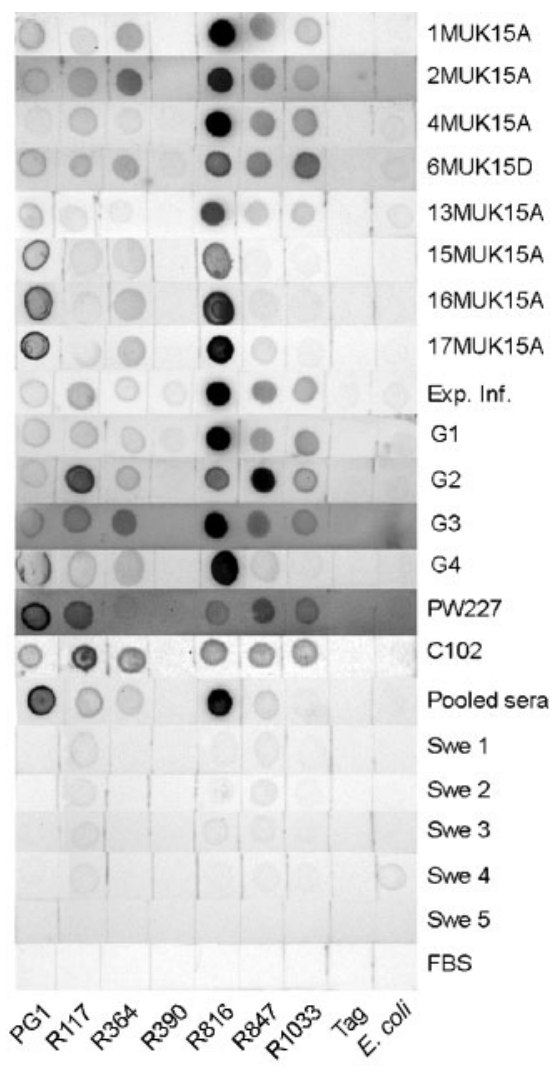

Fig. 1. Screening for immunoreactivity of the six Vmm-type proteins, here shown as dot blots of recombinant proteins and detected with 15 different bovine sera from CBPP-affected cattle, a pool of these sera, and six negative controls, including five healthy Swedish cattle and FBS. A representative blot from a total of three experiments is shown for each serum. Each membrane strip contained whole-cell lysate of $M$. mycoides SC strain $\mathrm{PG}^{\top}$ as a positive control, the recombinant proteins R117, R364, R390 (Vmm), R816, R847 and R1033, and the negative controls $\mathrm{His}_{6}-$ $\mathrm{ABP}$ (Tag) and E. coli whole-cell lysate. Binding of serum antibodies to a protein is indicated by a dark spot. Blots with sera $\mathrm{C} 102$, pooled sera and Swe 1-5 were made with a more concentrated $\mathrm{PG} 1^{\top}$ lysate than the other blots.

lysate was used, the $\mathrm{PG} 1^{\mathrm{T}}$ signal is stronger in comparison to Fig. 1.

To verify protein size and protein purity, and to identify the recombinant proteins, Western blot analyses were used. Each membrane was Ponceau S-stained to visualize total protein content followed by specific detection with one of the 15 CBPP sera, a His ${ }_{6}-\mathrm{ABP}$-directed antibody, control sera or secondary HRP-conjugated antibodies alone. A selection of blots is shown in Fig. 3 and all results are compiled in Table 3. The $\mathrm{His}_{6}-\mathrm{ABP}$-antibody staining showed clear and strong bands at the expected sizes of the recombinant proteins and for the $\mathrm{His}_{6}-\mathrm{ABP}$ control. Protein R117 had a second weak band of smaller size, indicating partially degraded protein or proteolytic fragments, which was further substantiated by the serum-detected blots that
Table 3. Summary of dot blot (DB) and Western blot (WB) results from screening the recombinant proteins R117, R364, $\mathrm{R} 390, \mathrm{R} 816, \mathrm{R} 847$ and R1033 with 15 CBPP-diseased bovine sera

Signal intensities in dots and bands: ++ , strongly positive; + , positive; $+/-$, weak or inconclusive; - , negative. The results were judged from three separate sets of dot blot and two separate Western blot experiments performed under standardized conditions with two different dilutions of sera.

\begin{tabular}{|c|c|c|c|c|c|c|c|}
\hline \multirow[t]{2}{*}{ Serum } & \multirow[t]{2}{*}{ Method } & \multicolumn{6}{|c|}{ Protein } \\
\hline & & R117 & R364 & R390 & R816 & R847 & R1033 \\
\hline \multirow[t]{2}{*}{ 1MUK15A } & DB & $+1-$ & + & - & ++ & + & + \\
\hline & WB & - & + & - & ++ & + & + \\
\hline \multirow[t]{2}{*}{ 2MUK15A } & DB & + & + & - & ++ & + & + \\
\hline & WB & + & + & - & ++ & + & + \\
\hline \multirow[t]{2}{*}{ 4MUK15A } & DB & + & $+1-$ & - & ++ & + & + \\
\hline & WB & + & + & $+1-$ & ++ & + & + \\
\hline \multirow[t]{2}{*}{ 6MUK15D } & DB & + & + & - & + & + & + \\
\hline & WB & + & + & $+1-$ & ++ & + & ++ \\
\hline \multirow[t]{2}{*}{ 13MUK15A } & DB & $+1-$ & $+1-$ & - & + & $+1-$ & $+1-$ \\
\hline & WB & $+1-$ & + & - & ++ & + & + \\
\hline \multirow[t]{2}{*}{ 15MUK15A } & DB & + & + & - & ++ & - & $+1-$ \\
\hline & WB & + & ++ & - & ++ & $+1-$ & + \\
\hline \multirow[t]{2}{*}{ 16MUK15A } & DB & $+1-$ & + & - & ++ & $+1-$ & $+1-$ \\
\hline & WB & $+1-$ & ++ & - & ++ & + & + \\
\hline \multirow[t]{2}{*}{ 17MUK15A } & DB & + & + & - & ++ & + & $+1-$ \\
\hline & WB & + & ++ & - & ++ & + & + \\
\hline \multirow[t]{2}{*}{ Exp. Inf. } & DB & + & $+1-$ & $+1-$ & ++ & + & + \\
\hline & WB & + & + & + & ++ & + & + \\
\hline \multirow[t]{2}{*}{ G1 } & DB & + & + & - & ++ & + & + \\
\hline & WB & + & + & - & ++ & + & + \\
\hline \multirow[t]{2}{*}{ G2 } & DB & ++ & + & - & + & ++ & + \\
\hline & WB & ++ & ++ & - & ++ & ++ & ++ \\
\hline \multirow[t]{2}{*}{ G3 } & DB & + & + & - & ++ & + & + \\
\hline & WB & + & + & - & ++ & + & + \\
\hline \multirow[t]{2}{*}{ G4 } & DB & + & + & - & ++ & + & $+1-$ \\
\hline & WB & + & ++ & - & ++ & + & + \\
\hline \multirow[t]{2}{*}{ PW227 } & DB & + & $+1-$ & - & + & + & + \\
\hline & WB & ++ & $+1-$ & $+1-$ & + & + & ++ \\
\hline \multirow[t]{2}{*}{ C102 } & DB & + & + & - & + & + & + \\
\hline & WB & + & + & - & + & + & ++ \\
\hline
\end{tabular}

also showed bands of smaller sizes. A comparison of $\mathrm{His}_{6}-$ $\mathrm{ABP}$ and Ponceau $\mathrm{S}$ stains showed that only small amounts of contaminating proteins were present, although protein R364 had several bands corresponding to E. coli proteins. There were no interactions with the $\mathrm{His}_{6}-\mathrm{ABP}$ tag in any of the Western blots. All five recombinant Vmm-type proteins generated bands with the CBPP sera but with different signal intensities and with variations between sera. The previously studied Vmm was the least immunoreactive protein, generating weak and therefore inconclusive bands with sera from three natural infections and a distinct band only in the experimental infection. Despite the small quantity of R816 on the membranes, it generated the strongest signal with most sera. It is noteworthy that the staining of the $M$. 


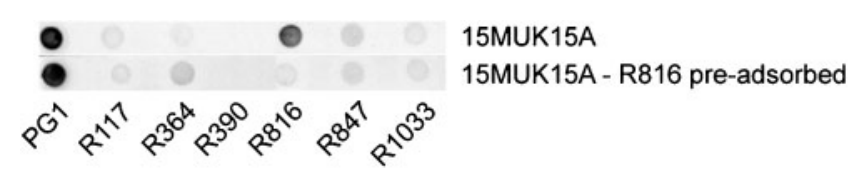

Fig. 2. Dot blots made for evaluation of specificity of the serum antibodies to the recombinant $\mathrm{Vmm}$-type proteins. The specific signal for a Vmm-type protein was blocked without affecting the other signals, demonstrated here with and without pre-adsorption with protein R816. The PG1 signal is stronger in comparison to Fig. 1 , as a more concentrated $P G 1^{\top}$ lysate was used.

mycoides SC lysate varied among the sera even with identical aliquots of the same lysate. Blots with the control sera from healthy bovines and secondary antibodies were blank or showed rare, extremely faint bands; hence, the noise levels in the disease serum blots were considered to be negligible.
Overall, the dot and Western blots gave similar results. MSC_816 and its homologues raised a strong humoral response for all tested CBPP sera, while IgGs binding Vmm were rare. Antibody levels to the other Vmm-type proteins showed more variation.

\section{Generation of Vmm-type protein-specific antibodies}

pAbs to the five recombinant proteins were generated in rabbits. In addition, the mouse mAb 5G1, which targets Vmm (Persson et al., 2002), was used. The rabbit sera were affinity-purified using the recombinant proteins as ligands to obtain monospecific polyclonal antisera. The crossreactivity of the polyclonal antisera was tested by dot blotting in duplicates. The six recombinant proteins, an $M$. mycoides SC strain $\mathrm{PG}^{\mathrm{T}}$ lysate and four recombinant proteins of human origin were analysed with the five

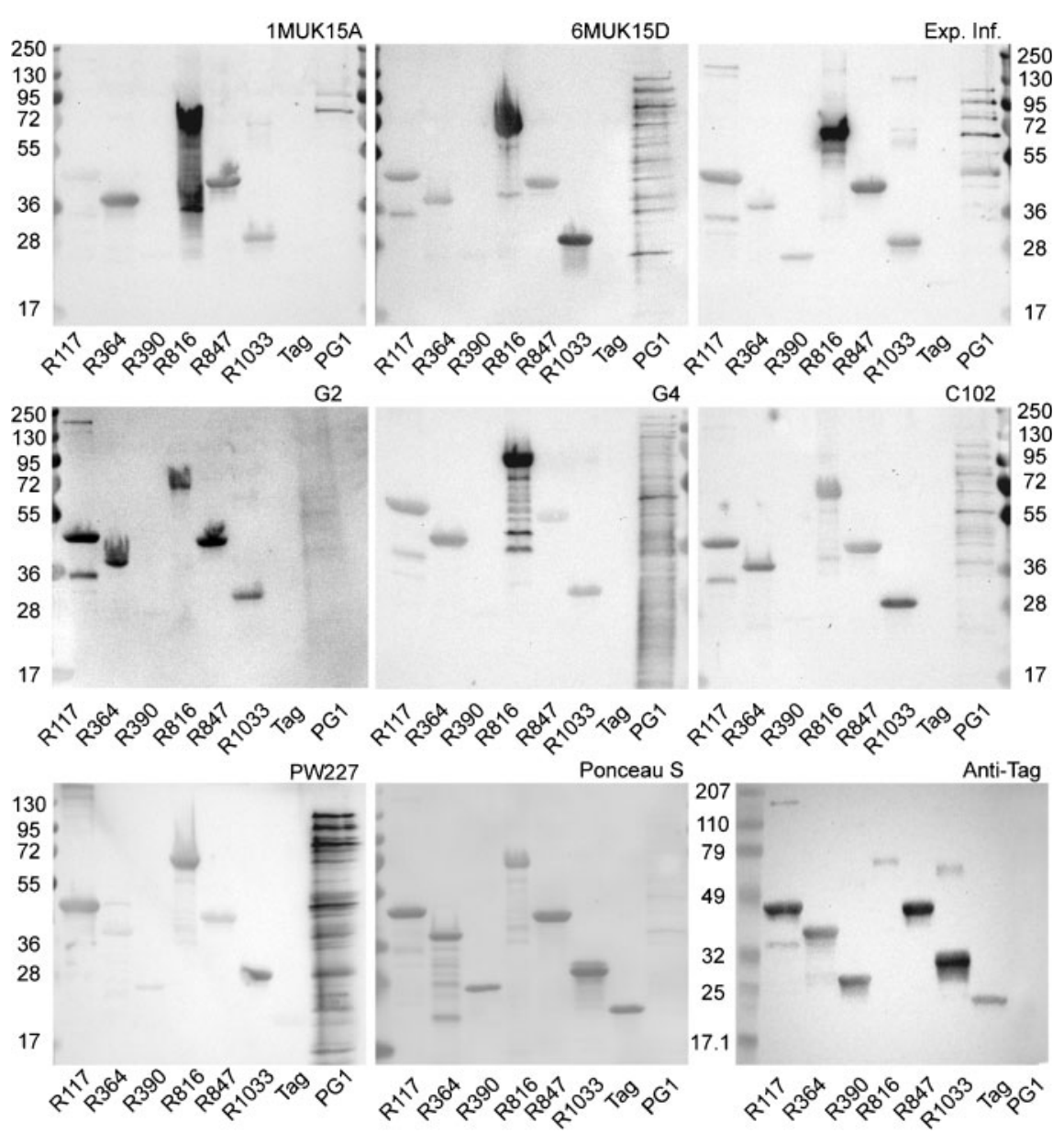

Fig. 3. Western blots of the recombinant proteins $R 117, \mathrm{R} 364, \mathrm{R} 390$ (Vmm), R816, R847 and R1033 to screen for immunoreactivity of the corresponding native proteins in $M$. mycoides SC infections. The blots were analysed with bovine sera from CBPP-affected cattle (1MUK15A, 6MUK15D, Exp. Inf., G2, G4, C102 and PW227). His 6 -ABP protein (Tag) and a whole-cell lysate of $M$. mycoides $S C$ strain $P G 1^{\top}$ were included as negative and positive controls, respectively. The last two blots were stained with Ponceau $S$ in order to visualize the total protein content on the membrane and with a Tag-specific antibody that identifies all recombinant proteins. 
affinity-purified pAbs and the 5G1 mAb (Fig. 4). All five antibodies against the recombinant proteins generated signals with their respective partner at 1:10 000 dilutions. A117, A816 and A847 generated weak cross-reactivity with each other. None of the specific pAbs stained the PG1 ${ }^{\mathrm{T}}$ lysate. The 5G1 staining showed strong specific staining of the recombinant Vmm (R390) and weak staining of the $\mathrm{PG1}^{\mathrm{T}}$ lysate. Furthermore, there was no cross-reactivity with the $\mathrm{His}_{6}-\mathrm{ABP}$ tag or the recombinant proteins of human origin produced using the same vector and methodology, which were used as negative controls. It is noteworthy that antibodies A816 and A1033 will probably bind all the homologues to proteins MSC_0816 and MSC_1033 mentioned in the 'Selection and in silico analysis of Vmm-type proteins' section above.

\section{Expression of Vmm-type proteins on in vitro cultured M. mycoides SC}

Colony blots of M. mycoides SC strains PG1 ${ }^{\mathrm{T}}$ and M223/90 were analysed with the five affinity-purified antibodies and $\mathrm{mAb} 5 \mathrm{G} 1$. Colonies that were positively stained entirely or in sectors were obtained for both strains with antibody A364 (Fig. 5), which confirms the bioinformatic prediction that protein MSC_0364 has variable expression. Approximately $20 \%$ of the colonies in M223/90 were stained, but only $5 \%$ in $\mathrm{PG}^{\mathrm{T}}$. Antibody A847 gave an inconclusive result in that one colony was positive among thousands of negatives in strain M223/90 only; however, this may be a result of very rare expression of MSC_0847 under in vitro conditions. Expression of MSC_0117, MSC_0816 and MSC_1033 could not be detected with antibodies A117, A816 or A1033 by colony blotting. The control experiment with mAb 5G1 showed a high frequency of variation in the expression of $\mathrm{Vmm}$ in $\mathrm{PG}^{\mathrm{T}}$, in accordance with previous results (Persson et al., 2002).

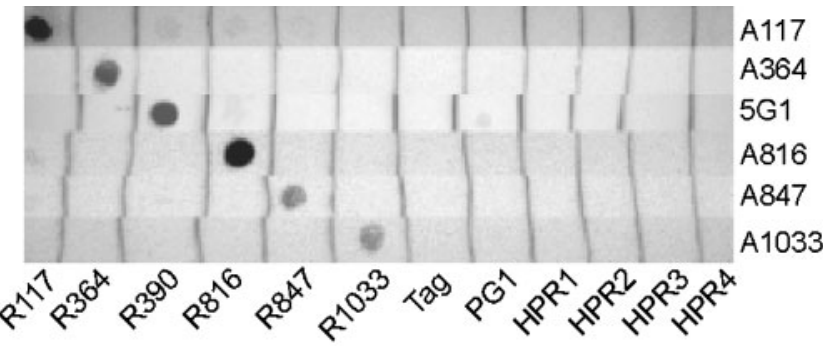

Fig. 4. Specificity of the affinity-purified pAbs. The dot blots contain the recombinant proteins R117, R364, R390, R816, R847 and $\mathrm{R} 1033, \mathrm{His}_{6}-\mathrm{ABP}$ (Tag), a whole-cell lysate of $M$. mycoides $\mathrm{SC}$ strain $\mathrm{PG} 1^{\top}$ and four similar recombinant proteins of human origin (HPR1-4) produced using the same vector, which were used as negative controls. Dot blot strips were immunostained with specific polyclonal antiserum A117, pAb A364, mAb 5G1, pAb $A 816, p A b A 847$ and pAb A1033. Specific binding of an antibody to its antigen is indicated by a dark spot.

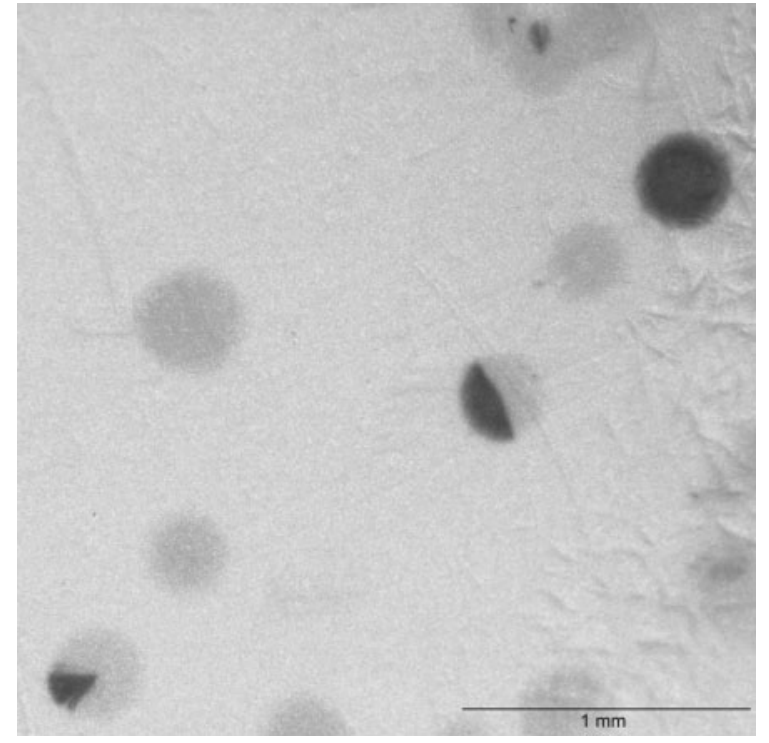

Fig. 5. Colony blotting with A364 of $M$. mycoides SC strain M223/90 showing variable expression of surface protein MSC_0364 within and between colonies. The black colour is specific antibody staining and indicates expression of MSC_0364, and the grey colour is Ponceau S staining of all proteins, showing all colonies.

To investigate whether the negative results of colony blotting were caused by lack of expression, epitope masking or failure of the antibodies, Western blotting was performed on $\mathrm{PG1}^{\mathrm{T}}$ lysates (Fig. 6). Antibody A364 generated a strong band of the correct size and two smaller bands that were considered to be noise, thus supporting the previous colony blotting results. Antibodies A117 and A847 gave a single band that would support the hypothesis that MSC_0117 and MSC_0847 were expressed, but the protein sizes were different from the theoretical sizes of the native proteins (see Table 2). Two distinct protein bands were obtained with A1033, indicating that both MSC_1033 and its homologue MSC_1058 were expressed. Even though the lower band corresponds to the theoretical size of MSC_1058 (15.7 kDa), it is tempting to assume that both proteins have retarded migration. No bands were obtained for antibody A816 despite the fact that this antibody should recognize five protein homologues. It is noteworthy that these Western blots contained a more concentrated $\mathrm{PGl}^{\mathrm{T}}$ lysate than the dot blots.

To further investigate the expression data, RT-PCR was performed on RNA extracted from a $\mathrm{PG1}^{\mathrm{T}}$ culture. Amplicons were obtained for all the Vmm-type proteins by RT-PCR but were not obtained by regular PCR on the same RNA template, showing that the transcripts were present in the cultured $P G 1^{\mathrm{T}}$. The presence of transcripts supported the findings for Vmm-type protein MSC_0364. For proteins MSC_0117 and MSC_0847, we conclude that the transcript had been detected, and it is likely that the 


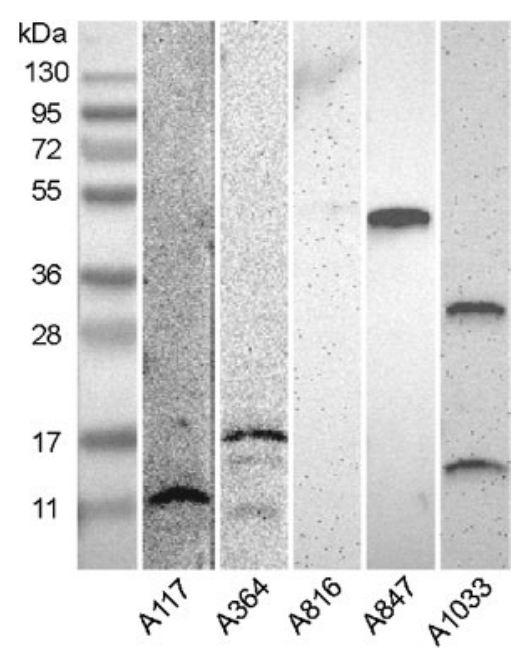

Fig. 6. Western blots of a whole-cell lysate of $M$. mycoides SC strain $P G 1^{\top}$ stained with antibodies $A 117, A 364, A 816, A 847$ and A1033.

bands obtained in the Western blots were the correct proteins; however, they had an atypical migration in SDS-PAGE, which may be accounted for by imprecise bioinformatic size predictions. The transcript for protein MSC_1033 and/or MSC_1058 was amplified; however, we have no method to differentiate them. This is also true for protein MSC_0816 and its homologues, although no protein expression at all could be detected despite multiple immunoblottings being performed under different conditions with cultured $\mathrm{PG} 1^{\mathrm{T}}$ and $\mathrm{M} 223 / 90$. Interestingly, this protein often gave the strongest signals when the recombinant proteins were immunoblotted and screened with bovine sera.

\section{DISCUSSION}

In this investigation, five Vmm-type proteins of $M$. mycoides SC and Vmm itself were analysed. The bioinformatic predictions identified signal peptides in all the proteins (five of which were prolipoproteins), suggesting surface location, and also identified promoter structures that indicate variable expression. A scheme for highthroughput cloning, mutagenesis, expression in E. coli and purification of recombinant $M$. mycoides SC proteins was developed as a tool to perform studies that may support and supplement the bioinformatic predictions. This scheme will be beneficial for further research on $M$. mycoides SC and can be used in studies on other mycoplasma species. This scheme yielded sufficient recombinant Vmm-type protein from $100 \mathrm{ml}$ of culture to perform Western and dot blotting experiments to assess the immunogenicity of the native Vmm-type proteins, to make antigens for antibody generation, and to make columns for affinity purification of the antisera.
When sera from natural CBPP infections were analysed for the presence of antibodies that target Vmm-type proteins, it was found that all sera contained antibodies to some or all of the Vmm-type proteins or their homologues. However, the sera generated different dot and Western blot patterns (Figs 1 and 3, Table 3), suggesting that the immune responses in the individual bovines were triggered by different proteins, either because this is the nature of the immune system or because the protein composition on the mycoplasma surface differed due to variable expression of each Vmm-type protein. For most sera, dot and Western blotting gave similar results, but we could observe differences in signal strength. This is expected due to the conformational differences of the proteins in the assays. Surprisingly, the previously studied Vmm (MSC_0390), which is thought to be immunodominant, generated the poorest IgG response and no response at all in most sera. Protein MSC_0816 and its homologues generated the strongest response overall. Interestingly, the differences in immunoreactivity between individual sera from one outbreak were as pronounced as the deviations seen between sera from different outbreaks in vastly separated geographical regions. This is clearly seen with the Botswana serum G2, which has a particular protein profile, whereas most Namibian and Botswana sera follow a more uniform pattern. The sera from Tanzania and Kenya gave distinct protein profiles; however, more sera must be analysed to find out if this is representative of the region or just specific for the individual bovines.

The dot and Western blotting results presented were repeated three times and twice, respectively, with high reproducibility. Standardized experimental conditions were used for each set of blots, with identical protein batches and volumes, and fixed serum dilutions. Signal intensities in Table 3 were annotated by judging all available replicates of the blots. It is not possible, however, to directly quantify the amounts of bound IgG antibodies by measuring the signal intensity when using an enhancedchemiluminescence detection system, since subtle changes will influence the signal strength, such as the amount of protein in the blot, the time for photo acquisition and the enzymic luminescence reaction. Clearly the light emitted from a strong positive signal will suppress the detection of a weak signal on the same blot. An example is the dot blot with serum PW227 in Fig. 1, where responses to the recombinant proteins are weak compared to the $P G 1^{\mathrm{T}}$ lysate response, which is why the $P G 1^{T}$ signal appears more intense than for blots in which one of the recombinants gives the predominant signal. Similarly, a negative control often generated a weak signal when no positive signal was present. For this reason signals were categorized as inconclusive when only weak signals were obtained compared to the controls, or if the higher dilution of a serum lacked the corresponding signal of a lower dilution. Furthermore, we do not know the effects of storage, transport, cycles of freezing and thawing, age, lyophilization, etc. on the immunoglobulins. Naturally, the quality of the disease sera 
will affect the general signal strength in an experiment, but should have less effect on the relative amounts of proteinspecific antibodies that cause the protein profile within one blotting membrane. The only fresh samples used in this study were from the five healthy control cattle, and it is possible that these five samples had a higher general IgG titre than those of the stored CBPP-positive sera.

Having concluded that the Vmm-type proteins or their homologues are expressed in natural infections and generate a humoral immune response, variable protein expression in vitro was also examined. All results for MSC_0364 were congruent and showed that this protein is expressed in a culture medium environment, and the colony immunostaining confirmed that its expression is variable. Transcripts of all Vmm-type proteins were identified by RT-PCR in total RNA of strain $P G 1^{\mathrm{T}}$, although this method is very sensitive and does not indicate transcription levels. Colony blots were essentially negative and therefore inconclusive. Western blots of PG1 ${ }^{\mathrm{T}}$ lysates provided some support for expression of the Vmmtype proteins, with one distinct band for A117 and A847, and two distinct bands for A1033, as expected. There is still some uncertainty regarding these results, since the detected bands were of sizes that differed from the calculated theoretical protein sizes. It is well known, however, that proteins and especially lipoproteins can display unpredictable migration in SDS-PAGE (Banker \& Cotman, 1972; Miyake et al., 1978; Simons \& Helenius, 1970). At this point one can only speculate whether the failure to detect proteins MSC_0117, MSC_847 and MSC_1033 in colony blots is due to epitope masking or a protein conformation problem that makes the antibodies unsuitable for assays with native, folded proteins, even if they worked well in Western blot applications and dot blotting of recombinant proteins. Using these techniques we were unable to determine if these three proteins are variably expressed. The last protein, MSC_0816, and its homologues could not be detected using any of the immunoassays.

An interesting aspect of the analyses was the deviation between our experimental observations and the expected expression profile of the Vmm-type proteins, as judged from the promoter sequences. Looking at the genomic sequence of $M$. mycoides SC strain $\mathrm{PGl}^{\mathrm{T}}$, most of these Vmm-type proteins would be expected to be silent in this strain according to the length of the promoter spacer. Only one of the expressed Vmm-type proteins, MSC_117, had a promoter spacer length of $17 \mathrm{bp}$ and should theoretically be expressed. Colony blotting indicated that MSC_364 and MSC_390 were expressed, while MSC_117 was not. One explanation would be that the genome sequence and the colony blots were made from different passages of PG1 ${ }^{\mathrm{T}}$. Furthermore, transcripts were detected for all the Vmmtype proteins, but the sensitivity of PCR would detect a very small fraction of expressed genes that may be undetectable with the other methods. Generally, comparisons between experiments and batches of cultures are unreliable when working with variable proteins.
It may be argued that the antigen repertoire of in vitrocultured strains of M. mycoides SC differs from those of strains in natural infections, since vaccination with nonviable in vitro cultivated strains often gives insufficient protection. Therefore, one hypothesis is that vaccine candidates should be searched for within the set of proteins that are expressed in the infected host but not in the laboratory. Theoretically, a vaccine of lysed whole cells spiked with a blend of all Vmm-type proteins should match all expression combinations in infected hosts.

Following the systematic analysis of surface proteins by recombinant technology we have presented results for six Vmm-type proteins that generated immune responses in vivo. We have also shown that the IgG response targets different Vmm-type proteins in individual sera. Further work using serial bleeds during a natural infection would increase our understanding of the natural variability in the expression of these proteins and possible infection-stagedependent variability. It is appealing to consider these Vmm-type proteins as potential components of a recombinant protein vaccine, although their use in a vaccine needs to be further evaluated, since a humoral immune response does not necessarily mean that these proteins raise a cell-mediated immune response or a protective immune response. A recombinant vaccine to M. mycoides SC would have several advantages over live attenuated vaccines, which include durability in storage and transport, fewer undesirable side effects, since it would be more defined, and most importantly in this case, there are more options to modulate a recombinant vaccine. For example, one can readily add or remove fusion partners to enhance host responses or make di- and multimers of the recombinant proteins in the vaccine.

The recombinant proteins and their corresponding specific antibodies produced in this study have the potential to be powerful reagents for future protein investigations such as ELISA development, immunohistochemistry and studies of protein interactions.

\section{ACKNOWLEDGEMENTS}

We would like to thank Gerrit Viljoen, International Atomic Energy Agency, Vienna, Baptiste Dungu, Ondertepoort Biological Products, Ondertepoort, South Africa, Otto Hübschle, Ministry of Agriculture, Water and Fishery, Windhoek, Namibia, and John B. March, BigDNA Ltd, Roslin, Scotland, for providing the bovine sera used in this study. We would also like to thank our colleagues Stefan Jernstedt, Agneta Stillesjö and Virginia Melys at the National Veterinary Institute (SVA) in Uppsala, Sweden, for assisting with the colony blotting. The study was funded by the Swedish International Development Cooperation Agency (SIDA).

\section{REFERENCES}

Abdo, E. M., Nicolet, J. \& Frey, J. (2000). Antigenic and genetic characterization of lipoprotein LppQ from Mycoplasma mycoides subsp. mycoides SC. Clin Diagn Lab Immunol 7, 588-595. 
Altschul, S. F., Madden, T. L., Schaffer, A. A., Zhang, J., Zhang, Z., Miller, W. \& Lipman, D. J. (1997). Gapped BLAST and PSI-BLAST: a new generation of protein database search programs. Nucleic Acids Res 25, 3389-3402.

Banker, G. A. \& Cotman, C. W. (1972). Measurement of free electrophoretic mobility and retardation coefficient of proteinsodium dodecyl sulfate complexes by gel electrophoresis. A method to validate molecular weight estimates. J Biol Chem 247, 5856-5861.

Bölske, G. (1988). Survey of mycoplasma infections in cell cultures and a comparison of detection methods. Zentralbl Bakteriol Mikrobiol Hyg [A] 269, 331-340.

Bölske, G., Msami, H. M., Gunnarsson, A., Kapaga, A. M. \& Loomu, P. M. (1995). Contagious bovine pleuropneumonia in northern Tanzania, culture confirmation and serological studies. Trop Anim Health Prod 27, 193-201.

Boonstra, E., Lindbaek, M., Fidzani, B. \& Bruusgaard, D. (2001). Cattle eradication and malnutrition in under five's: a natural experiment in Botswana. Public Health Nutr 4, 877-882.

Brocchi, E., Gamba, D., Poumarat, F., Martel, J. L. \& De Simone, F. (1993). Improvements in the diagnosis of contagious bovine pleuropneumonia through the use of monoclonal antibodies. Rev Sci Tech 12, 559-570.

Buttery, S. H., Lloyd, L. C. \& Titchen, D. A. (1976). Acute respiratory, circulatory and pathological changes in the calf after intravenous injections of the galactan from Mycoplasma mycoides subsp. mycoides. J Med Microbiol 9, 379-391.

Cheng, X., Nicolet, J., Miserez, R., Kuhnert, P., Krampe, M., Pilloud, T., Abdo, E. M., Griot, C. \& Frey, J. (1996). Characterization of the gene for an immunodominant $72 \mathrm{kDa}$ lipoprotein of Mycoplasma mycoides subsp. mycoides small colony type. Microbiology 142, 3515-3524.

Citti, C. \& Rosengarten, R. (1997). Mycoplasma genetic variation and its implication for pathogenesis. Wien Klin Wochenschr 109, 562-568.

Gaurivaud, P., Persson, A., Grand, D. L., Westberg, J., Solsona, M., Johansson, K. E. \& Poumarat, F. (2004). Variability of a glucose phosphotransferase system permease in Mycoplasma mycoides subsp. mycoides small colony. Microbiology 150, 4009-4022.

Hübschle, O. J., Ayling, R. D., Godinho, K., Lukhele, O., Tjipura-Zaire, G., Rowan, T. G. \& Nicholas, R. A. (2006). Danofloxacin (Advocin) reduces the spread of contagious bovine pleuropneumonia to healthy in-contact cattle. Res Vet Sci 81, 304-309.

Juncker, A. S., Willenbrock, H., Von Heijne, G., Brunak, S., Nielsen, H. \& Krogh, A. (2003). Prediction of lipoprotein signal peptides in Gramnegative bacteria. Protein Sci 12, 1652-1662.

Krogh, A., Larsson, B., von Heijne, G. \& Sonnhammer, E. L. (2001). Predicting transmembrane protein topology with a hidden Markov model: application to complete genomes. J Mol Biol 305, 567-580.

Kusiluka, L. J. \& Sudi, F. F. (2003). Review of successes and failures of contagious bovine pleuropneumonia control strategies in Tanzania. Prev Vet Med 59, 113-123.

Larsson, M., Gräslund, S., Yuan, L., Brundell, E., Uhlén, M., Höög, C. \& Ståhl, S. (2000). High-throughput protein expression of cDNA products as a tool in functional genomics. J Biotechnol 80, 143-157.

Le Grand, D., Solsona, M., Rosengarten, R. \& Poumarat, F. (1996). Adaptive surface antigen variation in Mycoplasma bovis to the host immune response. FEMS Microbiol Lett 144, 267-275.

Libon, C., Corvaia, N., Haeuw, J. F., Nguyen, T. N., Ståhl, S., Bonnefoy, J. Y. \& Andreoni, C. (1999). The serum albumin-binding region of streptococcal protein $\mathrm{G}(\mathrm{BB})$ potentiates the immunogenicity of the G130-230 RSV-A protein. Vaccine 17, 406-414.

March, J. B. (2004). Improved formulations for existing CBPP vaccines - recommendations for change. Vaccine 22, 4358-4364.
Mbulu, R. S., Tjipura-Zaire, G., Lelli, R., Frey, J., Pilo, P., Vilei, E. M., Mettler, F., Nicholas, R. A. \& Huebschle, O. J. (2004). Contagious bovine pleuropneumonia (CBPP) caused by vaccine strain T1/44 of Mycoplasma mycoides subsp. mycoides SC. Vet Microbiol 98, 229-234.

Miles, R. J., Taylor, R. R. \& Varsani, H. (1991). Oxygen uptake and $\mathrm{H}_{2} \mathrm{O}_{2}$ production by fermentative Mycoplasma spp. J Med Microbiol 34, 219-223.

Miyake, J., Ochiai-Yanagi, S., Kasumi, T. \& Takagi, T. (1978). Isolation of a membrane protein from $R$. rubrum chromatophores and its abnormal behavior in SDS-polyacrylamide gel electrophoresis due to a high binding capacity for SDS. J Biochem (Tokyo) 83, 1679-1686.

Monnerat, M. P., Thiaucourt, F., Poveda, J. B., Nicolet, J. \& Frey, J. (1999). Genetic and serological analysis of lipoprotein LppA in Mycoplasma mycoides subsp. mycoides LC and Mycoplasma mycoides subsp. capri. Clin Diagn Lab Immunol 6, 224-230.

Nicholas, R. A. \& Bashiruddin, J. B. (1995). Mycoplasma mycoides subspecies mycoides (small colony variant): the agent of contagious bovine pleuropneumonia and member of the "Mycoplasma mycoides cluster". J Comp Pathol 113, 1-27.

Nicholas, R., Bashiruddin, J., Ayling, R. \& Miles, R. (2000). Contagious bovine pleuropneumonia: a review of recent developments. Vet Bull 70, 827-838.

Nielsen, H. \& Krogh, A. (1998). Prediction of signal peptides and signal anchors by a hidden Markov model. Proc Int Conf Intell Syst Mol Biol 6, 122-130.

Nielsen, H., Engelbrecht, J., Brunak, S. \& von Heijne, G. (1997). Identification of prokaryotic and eukaryotic signal peptides and prediction of their cleavage sites. Protein Eng 10, 1-6.

Nilsson, P., Paavilainen, L., Larsson, K., Odling, J., Sundberg, M., Andersson, A. C., Kampf, C., Persson, A., Al-Khalili Szigyarto, C. \& other authors (2005). Towards a human proteome atlas: highthroughput generation of mono-specific antibodies for tissue profiling. Proteomics 5, 4327-4337.

Nygren, P. Å., Eliasson, M., Abrahmsen, L., Uhlén, M. \& Palmcrantz, E. (1988). Analysis and use of the serum albumin binding domains of streptococcal protein G. J Mol Recognit 1, 69-74.

Persson, A., Jacobsson, K., Frykberg, L., Johansson, K. E. \& Poumarat, F. (2002). Variable surface protein Vmm of Mycoplasma mycoides subsp. mycoides small colony type. J Bacteriol 184, 3712-3722.

Pilo, P., Martig, S., Frey, J. \& Vilei, E. M. (2003). Antigenic and genetic characterisation of lipoprotein lppC from Mycoplasma mycoides subsp. mycoides SC. Vet Res 34, 761-775.

Porath, J., Carlsson, J., Olsson, I. \& Belfrage, G. (1975). Metal chelate affinity chromatography, a new approach to protein fractionation. Nature 258, 598-599.

Provost, A. (1996). Strategies for prevention and eradication of contagious bovine pleuropneumonia with or without vaccination. Rev Sci Tech 15, 1355-1371 (in French).

Quevillon, E., Silventoinen, V., Pillai, S., Harte, N., Mulder, N., Apweiler, R. \& Lopez, R. (2005). InterProScan: protein domains identifier. Nucleic Acids Res 33, W116-W120.

Razin, S., Yogev, D. \& Naot, Y. (1998). Molecular biology and pathogenicity of mycoplasmas. Microbiol Mol Biol Rev 62, 1094-1156.

Sachse, K., Helbig, J. H., Lysnyansky, I., Grajetzki, C., Muller, W., Jacobs, E. \& Yogev, D. (2000). Epitope mapping of immunogenic and adhesive structures in repetitive domains of Mycoplasma bovis variable surface lipoproteins. Infect Immun 68, 680-687.

Simons, K. \& Helenius, A. (1970). Effect of sodium dodecyl sulphate on human plasma low density lipoproteins. FEBS Lett 7, 59-63. 
Sjölander, A., Nygren, P. Å., Ståhl, S., Berzins, K., Uhlén, M., Perlmann, P. \& Andersson, R. (1997). The serum albumin-binding region of streptococcal protein G: a bacterial fusion partner with carrier-related properties. J Immunol Methods 201, 115-123.

Steen, J., Uhlen, M., Hober, S. \& Ottosson, J. (2006). Highthroughput protein purification using an automated set-up for high-yield affinity chromatography. Protein Expr Purif 46, 173-178.

Thiaucourt, F., Lorenzon, S., David, A., Tulasne, J. J. \& Domenech, J. (1998). Vaccination against contagious bovine pleuropneumonia and the use of molecular tools in epidemiology. Ann N Y Acad Sci 849, 146-151.

Thiaucourt, F., Yaya, A., Wesonga, H., Hübschle, O. J., Tulasne, J. J. \& Provost, A. (2000). Contagious bovine pleuropneumonia. A reassessment of the efficacy of vaccines used in Africa. Ann N Y Acad Sci 916, 71-80.

Tryon, V. V. \& Baseman, J. B. (1992). Pathogenic determinants and mechanisms. In Mycoplasmas: Molecular Biology and Pathogenesis, pp. 457-472. Edited by J. Maniloff, R. N. McElhaney, L. R. Finch \& J. B. Baseman. Washington DC: American Society for Microbiology.

Vilei, E. M., Abdo, E. M., Nicolet, J., Botelho, A., Goncalves, R. \& Frey, J. (2000). Genomic and antigenic differences between the European and
African/Australian clusters of Mycoplasma mycoides subsp. mycoides SC. Microbiology 146, 477-486.

Washburn, L. R., Hirsch, S. \& Voelker, L. L. (1993). Mechanisms of attachment of Mycoplasma arthritidis to host cells in vitro. Infect Immun 61, 2670-2680.

Westberg, J., Persson, A., Holmberg, A., Goesmann, A., Lundeberg, J., Johansson, K. E., Pettersson, B. \& Uhlén, M. (2004). The genome sequence of Mycoplasma mycoides subsp. mycoides SC type strain $\mathrm{PGl}^{\mathrm{T}}$, the causative agent of contagious bovine pleuropneumonia (CBPP). Genome Res 14, 221-227.

Windsor, R. S. (2000). The eradication of contagious bovine pleuropneumonia from south western Africa. A plan for action. Ann N Y Acad Sci 916, 326-332.

Windsor, R. S. \& Wood, A. (1998). Contagious bovine pleuropneumonia. The costs of control in central/southern Africa. Ann N Y Acad Sci 849, 299-306.

Zdobnov, E. M. \& Apweiler, R. (2001). InterProScan - an integration platform for the signature-recognition methods in InterPro. Bioinformatics 17, 847-848.

Edited by: C. Citti 